\title{
Que nous renseignent la modalisation et les rôles argumentatifs sur l'état de doute? Analyse d'une discussion philosophique avec des élèves de $\mathrm{CM} 2$
}

\author{
Anda, Fournel \\ Laboratoire Lidilem, Université Grenoble-Alpes, France \\ anda.fournel@gmail.com
}

\begin{abstract}
Résumé. L'objectif de cette étude est d'observer les conduites langagières et conceptuelles des élèves qui, engagés dans un dialogue philosophique entre pairs, sont amenés à argumenter et à problématiser. Ils parviennent à remettre en doute les opinions des autres ou leurs propres opinions afin de construire des points de vue. En fonction de la situation d'énonciation ou de co-énonciation, et du type d'activité menant au doute, les points de vue peuvent être justifiés ou pas encore explicités, ils peuvent s'opposer à ceux des autres ou encore être nuancés ou suspendus. Notre contribution commence par donner le cadre général dans lequel s'inscrit la discussion philosophique analysée ainsi que le contexte de sa réalisation. Elle esquisse par la suite les repères théoriques et les choix méthodologiques permettant de proposer un modèle d'analyse du doute qui fait appel aux actes philosophiques, à la modalisation et aux rôles actantiels argumentatifs (Proposant, Opposant et Tiers). Des extraits du corpus illustrent les propos. Nous envisageons ce modèle comme un outil pour explorer les conditions d'expression du doute dans une discussion philosophique et dégager des critères nécessaires pour distinguer un dialogue philosophique d'un débat argumentatif. Le modèle devra être testé sur d'autres discussions afin d'être ajusté et validé.
\end{abstract}

\begin{abstract}
The aim of this study is to observe linguistic and conceptual behaviors when students, engaged in a philosophical dialog between peers, argue and problematize. They call into question their own opinions or what others think in order to build points of view. Depending on the utterance or the co-utterance situation, and on type of activity leading to the doubt, the points of view can be justified, not yet clarified, opposite to others views, resumed or suspended. We start by giving the general framework in which fits the philosophical discussion analyzed as well as the context of its realization. Then we outline our theoretical directions and methodological choices enabling to propose an analysis model of the doubt using philosophical acts, modality and the "three-roles interaction" (Proponent, Opponent and Third party) in an argumentative situation. Finally we illustrate our hypothesis with extracts from the corpus. This model could be used to explore how the expression of doubt occurs. It could also provide criteria to distinguish a philosophical dialog from an argumentative debate. The model should be tested with other discussions in order to be adjusted and validated.
\end{abstract}




\section{Introduction}

L'objectif de cette étude est d'observer, à travers une analyse des conduites langagières, comment des élèves engagés dans un dialogue philosophique remettent en question, en partie ou en totalité, leurs propres opinions ou les opinions des autres. Celles-ci constituent la matière pour construire par la suite des points de vue justifiés. Il y a un enjeu pédagogique fort dans le développement de cette attitude chez les élèves : la remise en question aide à la compréhension et la construction du savoir (par le processus d'infirmation ou de validation des opinions), à la formation du jugement autonome et de l'esprit critique, à la collaboration entre les interactants engagés dans la résolution commune d'un problème. A travers le dialogue philosophique il ne s'agit pas de convaincre, comme dans un débat où chacun devient le défendeur d'une position, mais de co-construire la solution (Sasseville \& Gagnon, 2007 : 111). Autrement dit, c'est le processus qui est premier non pas le résultat. Ce sont les traits essentiels d'une démarche philosophique en communauté de recherche (Lipman, 2006 et Tozzi, 2007).

Notre contribution porte sur l'analyse de l'activité de douter à partir des données verbales dialogiques extraites de l'enregistrement vidéo d'une démonstration de discussion philosophique en communauté de recherche. Celle-ci s'est tenue à l'Unesco, lors des journées consacrées annuellement aux nouvelles pratiques philosophiques, avec des élèves de CM2 de l'école de La Source de Meudon (92), en novembre 2014. Le point de départ de la discussion est une question, proposée par l'animateur Michel Tozzi, professeur émérite et didacticien de la philosophie, qu'il s'agissait d'explorer collectivement : Pourquoi on dit "c'est pas juste »? La discussion se divise en deux parties : la première est une démarche de justification dans laquelle les élèves formulent tour à tour des exemples et des raisons ; la deuxième comporte un exercice de mise en situation autour du partage d'un gâteau à faire de la manière la plus juste. Nous définissons ainsi d'emblée deux éléments qui caractérisent cette discussion : argumentation et problématisation.

L'analyse s'appuie sur la notion centrale de $P D V$, défini comme «l'événement constitué par l'apparition d'un énoncé » (Ducrot, 1984), qualifiée d'instable surtout lorsqu'il est articulé en contexte avec la polyphonie (Rabatel, 2003). Différentes formes de PDV peuvent être identifiées selon des situations d'énonciation ou de co-énonciation : le PDV non encore explicité (Vion, 2012), le PDV justifié d'emblée, le PDV qui s'oppose, le PDV repris et éventuellement nuancé, enfin le PDV suspendu. Si le choix d'un PDV ou d'un changement de PDV renvoie essentiellement à des stratégies énonciatives de la part des participants (Rabatel, 2003), nous faisons l'hypothèse que le statut ontologique du PDV est lié directement à la posture argumentative/problématisante des actants. Cette hypothèse nous semble d'autant plus appropriée à tester que la discussion philosophique à analyser s'appuie ouvertement sur une démarche de justification et de problématisation du concept de " juste ». Interviennent alors également les notions de proposition, d'opposition et de mise en doute reliées directement aux trois rôles actantiels argumentatifs : Proposant, Opposant et Tiers (Plantin, 2005).

Enfin, dans une approche interactionnelle et énonciative, le moment de suspension du jugement coïncide avec une non-prise en charge de l'énonciation par le locuteur (ibid.). L'effacement énonciatif ou, au contraire, la prise en charge énonciative, sont généralement mesurés à travers la modalisation entendue comme attitude du locuteur vis-à-vis de sa production langagière. Un rapport de proportionnalité inverse a été établi entre les deux éléments : plus le locuteur prend de la distance par rapport à l'opinion exprimée, 
via la modalisation, moins il assume le PDV qui s'y trouve exprimé et inversement, moins il se distancie du contenu de son énoncé plus la prise en charge du PDV est grande (Dubois et al., 1973). Néanmoins, sous un angle dialogique et pragmatique, ce rapport peut paradoxalement à la fois permettre et empêcher la prise en charge de l'énoncé, comme le souligne Vion (2012). Le locuteur peut prendre en charge, pour des raisons de communication et non pas par volonté, des inter-discours non-explicités car ceux-ci participent au sens de son énonciation, alors qu'en réalité il ne les assume pas complètement à cause de leur caractère implicite.

Cette étude commence par donner le cadre général dans lequel s'inscrit l'exemple de pratique de discussion philosophique analysée et le contexte de sa réalisation. Elle esquisse par la suite les repères théoriques et les choix méthodologiques permettant de proposer une modèle d'analyse philosophique et linguistique du doute, à travers notamment les actes philosophiques, la modalisation présente dans les conduites langagières et les rôles actantiels argumentatifs dans le discours. Elle se termine avec des illustrations extraites du corpus. Nous faisons le pari que le modèle permet d'explorer les conditions de l'expression du doute dans une discussion philosophique afin de comprendre comment le doute est associé dans le discours à une démarche à la fois argumentative et problématisante. Le modèle devra par la suite être testé, ajusté et stabilisé par son application à d'autres discussions, voire permettre de distinguer un débat argumentatif d'un dialogue philosophique.

\section{Cadre et contexte}

\subsection{La philosophie pour les enfants}

Recherche fondée sur la coopération et le dialogue, ayant comme visée le développement des compétences langagières et réflexives, la pratique de la philosophie avec les enfants s'inscrit dans un large mouvement éducatif nommé «Philosophy for Children », conçu et initié dans les années 70 par Matthew Lipman. Le questionnement et la pensée à la fois critique, créative et attentive se trouvent au cœur de ce programme d'apprentissage d'une pensée autonome et authentique. Ainsi une place importante est faite au doute et aux problèmes, qui s'intègre parfaitement dans la démarche de recherche de sens. "Questionner - soulignait l'auteur dans son ouvrage fondateur Thinking in Education (2003) c'est institutionnaliser et légitimer le doute et inviter à une évaluation critique. [...] La question apporte le doute dans les esprits et le doute marque le début de la recherche. " (Lipman, 2006 : 103). La conversation étant la façon la plus naturelle pour l'enfant de communiquer, il s'agit pour lui, outre l'écoute et l'attention développées au contact d'autrui, de questionner le sens (meaning) en prêtant de plus en plus attention aux relations que le langage entretient avec le monde. D'où une perspective interactionnelle de co-construction qui se dégage grâce à l'accomplissement d'une activité langagière collaborative (Mondada, 1999) appelée dans le mouvement éducationnel cité «communauté de recherche ».

\subsection{Un dispositif pédagogique français : la DVDP}

En France, ce dispositif pédagogique s'expérimente en milieu scolaire depuis plus de 20 ans. Dans sa version proposée par Michel Tozzi, Alain Delsol et Sylvain Connac, il s'agit à la fois d'un exercice démocratique et philosophique, DVDP signifiant discussion à visée démocratique et philosophique. Démocratique car, inspiré par la pédagogie coopérative, il met en place une répartition de rôles entre les élèves (président de séance, reformulateur, synthétiseur, discutants, observateurs...), des règles de prise de parole et une «éthique discussionnelle » (Tozzi, 2015). Philosophique par les exigences intellectuelles visées et développées dans la discussion, en nombre de trois : la problématisation, l'argumentation et la conceptualisation. L'animateur accompagne la mise en place et le développement de chacune de ces compétences en invitant les élèves à définir, distinguer, etc. pour conceptualiser ; justifier les points de vue exprimés ou élaborer des objections, etc. pour argumenter ; enfin formuler des questions philosophiques, complexifier, etc. pour problématiser. 
Au cœur de ce dispositif se trouvent deux principes fondamentaux interdépendants qui font qu'une discussion soit philosophique et démocratique. Il s'agit d'abord de la coopération qui est donnée à la fois par la co-animation solidaire de la discussion via les rôles complémentaires (précisés ci-dessus) et par la coopération sur le fond, renvoyant à une recherche commune, lorsque par exemple un élément apporté par un élève est relayé par un autre élève ou par plusieurs. La confrontation est vécue elle aussi sur le mode coopératif en vertu du fait que la remise en question ou les objections portent sur les idées et non pas sur les individus (Tozzi, id.). D'où le deuxième principe qui est celui d'une nécessité rationnelle de fonder la validité de son propos et de déconstruire les réponses toujours trop vite données » (Tozzi, 2007)».

\section{Ancrage théorique}

\subsection{Une conception pragmatiste du doute}

Le cadre théorique qui permet de circonscrire la problématique du doute nous situe d'abord dans une démarche pragmatiste, telle qu'elle a été mise en évidence par Tiercelin, dans un ouvrage qui met le doute lui-même en question (2005). L'auteur souligne l'originalité de deux conceptions, clairement anticartésiennes et anti-sceptiques : celle de Peirce (1877) axée sur le rapport entre le doute et la croyance et celle de Wittgenstein (1987), davantage axée sur la certitude. Pour ces auteurs, le doute s'appuie forcément sur des croyances, ainsi on peut exiger au doute, au même titre qu'à la certitude, des raisons. Si l'on accepte que certaines choses doivent être douteuses pour que d'autres ne le soient pas, il devient donc nécessaire de justifier le doute.

Peirce fera du doute la raison d'être de toute pensée : « la seule justification du raisonnement, c'est de calmer le doute » (1958, vol.1 : 166). Le sémiologue et philosophe américain inscrit le doute dans la démarche générale de la recherche, en l'abordant tour à tour sous l'angle épistémique, psychologique et logique. Il observe d'abord que le doute et la croyance sont « deux états d'esprit au sentir différent [...] la même différence que celle qu'il y a entre le rouge du bleu ou le plaisir de la douleur» (id. vol 1, 172). Mais le sentiment n'étant pas suffisant, une deuxième différence, cette fois pratique, peut être observée entre une habitude qui détermine nos actions et une absence d'habitude correspondant au moment du doute (id., vol.1 : 221). Enfin il évoque, en parlant du doute, un inconfort épistémique, « un état de malaise et insatisfaction dont on cherche, en luttant, à se libérer pour atteindre l'état de croyance » (id. vol.1 : 166).

Cette description a été reprise dans la tradition pragmatiste inaugurée après Peirce par Dewey (1910, 1997) et poursuivie par Lipman (2003). La pensée réflexive et chacune de ses opérations réfléchies se caractérisent par deux traits essentiels : d'abord un état de perplexité, d'hésitation ou de doute suivie d'une activité représentée par des recherches dirigées vers la découverte d'autres faits qui servent à confirmer ou infirmer l'opinion suggérée, dans un processus de validation.

\subsection{La visée éducative du doute}

Peirce et Dewey ont fortement influencé la conception de " communauté de recherche philosophique » introduite par Lipman dans le champ de l'éducation. En s'inspirant de ses prédécesseurs, Lipman développe une méthode pour éduquer la pensée. Il s'agit d'organiser une discussion dans laquelle les élèves cherchent ensemble une solution, grâce à une démarche qui permet d'éliminer au fur et à mesure, grâce à une activité critique, les raisonnements non pertinents (2003). Pour lutter contre toute paralysie de la pensée et afin de développer réflexion, approfondissement et pensée authentique, Lipman souligne la nécessité de "prévoir un espace disponible pour ce qui peut faire l'objet de doutes ou de problèmes » (2006 : 34). Le doute est « institutionnalisé et légitimé » par l'acte de questionner. L'auteur reprend la description de l'acte de pensée proposée par Dewey, pour présenter l'acte de questionner en deux mouvements : «la question apporte le doute dans les esprits» et «le doute marque le début de la recherche $(2006$ : 103). Le questionnement devient ainsi fondamental en éducation car il permet à la fois une évaluation critique des opinions et l'ouverture vers une recherche collaborative dans laquelle la place 
de la critique (rationalité) est pondérée par celle de la créativité (subjectivité) et du souci de l'autre (intersubjectivité). Il en résulte un nouvel concept défendu par Lipman : la « raisonabilité ».

On retrouve chez Tozzi la même visée éducative accordée au doute à travers deux des compétences philosophiques visées par la pratique des discussions philosophiques de type DVDP : la problématisation et l'argumentation. La première est définie comme une « une (auto) interrogation sur ses certitudes et celles d'autrui », alors que l'argumentation est érigée au rang de " nécessité rationnelle de fonder la validité de son propos et de déconstruire les réponses toujours trop vite données. » (Tozzi, 2007).

Observons que l'activité argumentative à visée éducative consiste à fonder et à objecter, dans un mouvement de confrontation entre les idées et pas entre les personnes. En cela, elle se distingue nettement de toute démarche argumentative de type rhétorique. Et pourtant est-il possible, dans un contexte interactionnel, pragmatique et ouvert à la négociation, d'isoler dans l'activité d'argumentative l'élément rationnel (logos) sans prêter attention à l'éthos (subjectivité) et au pathos (intersubjectivité) ? Certainement pas, et un des points à questionner dans une telle approche éducative rationnaliste est le caractère universel de l'auditoire implicitement visé.

\subsection{Le doute dans les théories argumentatives}

Les recherches actuelles sur l'argumentation mettent l'accent sur l'importance du dialogue dans l'activité argumentative, modélisée comme des positions contradictoires en interaction. L'argumentation est envisagée sur cette base également dans ses dimensions interpersonnelles (pathos) et subjectives (éthos). L'éthos, par exemple, renvoie à la polyphonie et donc à la construction et prise en charge d'un PDV (Plantin, 2015).

Le déclencheur de l'activité argumentative est la mise en doute d'un PDV. » (Plantin, 2005 : 52). Mais mettre en doute et douter semblent se distinguer comme étant des actes sous-tendus par des rôles argumentatifs distincts : d'une part remettre en question une proposition revient à s'y opposer, alors que douter suppose de sortir d'une confrontation directe de type opposition-proposition pour finalement suspendre tout assentiment à une proposition, qu'on la rejette ou qu'on l'envisage à titre d'hypothèse. " La communication est pleinement argumentative lorsque cette différence [opposition discours/contrediscours] est problématisée en une Question, et que se dégagent nettement les trois rôles actanciels de Proposant, d'Opposant et de Tiers. » (Plantin, 2005 :63). Finalement le rôle joué dans le dialogue par celui qui doute (le Tiers) va graduellement de l'ignorant, qui « ne sait pas », vers le « sans opinion », pour arriver enfin à celui qui, en voulant se prononcer en état de cause, suspend son assentiment.

\subsection{Les différents sens du doute}

Nous pouvons ainsi définir trois types de situation dans lesquelles le doute intervient : lorsqu'il y a hésitation dans l'expression d'un PDV d'un locuteur, impliquant une modalité de possibilité ou d'incertitude; lorsqu'il y a réaction ou remise en question d'un PDV exprimé par un interlocuteur (critique, restriction, désaccord) ou par le locuteur lui-même (autocritique); et enfin lorsqu'un locuteur refuse de donner son assentiment à un PDV, il suspend donc son jugement et garde le doute ouvert (problématise). Ces trois distinctions seront présentes dans l'analyse proposée ci-après, dans l'objectif de donner une perspective nuancée de l'expression du doute : soit comme indicateur d'incertitude (D1), soit comme un moyen argumentatif (D2) soit comme un outil de problématisation (D3). 


\section{Choix méthodologiques}

\subsection{Comment observer le doute?}

Comment observer le doute, cet état d'esprit qui n'est pas visible directement voire même impossible à observer lorsqu'il se passe uniquement dans la tête d'un locuteur ? Une façon de faire consiste à regarder les paroles et les autres comportements langagiers qui accompagnent cet état d'esprit. Lorsqu'un participant dit, en présageant cet état, « je doute », « je ne suis pas sûr », « je ne sais pas trop », «il se pourrait », « peut-être », «ça dépend » etc., il donne des indices, mais en aucun cas des preuves qu'il est en train de douter. Nous pouvons également prêter attention à la manière dont un locuteur exprime ses idées, ainsi lorsqu'il hésite (éprouve un embarras dans l'élocution, réalise des ratures orales) ou devient silencieux, où lorsqu'il envisage ses idées sous la forme d'une hypothèse ou d'une possibilité, il est possible qu'il soit dans un état de doute. Une autre façon d'examiner le doute, et c'est peut-être d'ailleurs le cas le plus fréquent, est d'observer l'absence de certitudes. Mais cela pourrait faire l'objet d'une autre analyse laquelle doit pouvoir aborder frontalement les certitudes. La modalisation dans la langue est par conséquent un excellent outil d'observation du rapport qu'entretien le locuteur avec sa propre production, des positions énonciatives qu'il prend vis-à-vis des opinions qu'il met en scène (Vion, 2012) ainsi que du rapport qu'il négocie ainsi avec ses co-énonciateurs (De Nuchèze \& Colletta, 2002). Enfin, l'intonation, les mimiques et les gestes complèteraient le panel de ressources à exploiter mais nous avons choisi de nous intéresser ici uniquement aux ressources verbales.

\subsection{Orientation des recherches vers les sciences cognitives}

Certaines recherches se sont attaquées directement aux processus cognitifs à l'œuvre dans l'activité de douter. Nous pouvons évoquer ici les travaux réalisées en sciences de l'éducation, renforcés par à l'avènement de la neuro-éducation autour de l'étude des croyances et du changement conceptuel. Le courant de l'épistémologie personnelle, avec les travaux de Perry, Kitchener, Kuhn et autres (Crahay, M. \& Fagnant, A., 2007), a influencé nombre de recherches appliquées aux apprentissages comme ce fut le cas des recherches récentes en neurosciences. Certaines se sont penchées, par exemple, sur le rôle du doute dans les apprentissages scientifiques qui nécessitent des changements conceptuels (Potvin, P., Masson, S. et Riopel, R., 2009). Ces phénomènes se produisent lorsque les élèves parviennent à surmonter les difficultés d'apprentissage liées à leur adhésion initiale à des conceptions spontanées, nonscientifiques, des phénomènes naturels. En essayant de comprendre comment ces conceptions évoluent et se développent, y compris en contexte de classe, ces études ont réussi à montrer que la fonction cognitive d'inhibition (à laquelle s'apparente le doute) est indispensable pour avoir des changements conceptuels réussis.

Ces études rejoignent celles développées par Houdé et son équipe (2014) sur le terrain de la psychologie expérimentale du développement de l'enfant, à partir de la théorie du double système pour expliquer le raisonnement humain. En s'intéressant aux biais de raisonnement, là où les conflits deviennent mesure du doute, ses études soulignent l'importance de l'inhibition comme facteur positif qui vient réguler les deux systèmes de pensée à l'œuvre chez l'être humain: la pensée intuitive ou automatique et la pensée réfléchie ou logico-mathématique. Selon l'auteur de la théorie de la résistance cognitive, dans une perspective anatomique, le système inhibiteur, situé au niveau du cortex préfrontal, est la région du cerveau qui se développe le plus tardivement et le plus lentement (à partir de 12 mois et elle dure jusqu'à l'âge adulte), raison pour laquelle les enfants, comme les adultes, ont des difficultés à inhiber. D'où une donnée fondamentale pour l'éducation préconisant l'instauration de l'apprentissage de l'inhibition dans les programmes scolaires.

Très récemment, une autre étude canadienne a mis en lumière le rôle de l'incertitude pour favoriser le changement conceptuel, toujours en lien avec l'apprentissage des notions scientifiques (Turmel, 2012). L'étude tend à démontrer, avec de l'imagerie cérébrale à l'appui, que mettre l'apprenant dans des conditions de doute intellectuel constituerait un levier d'apprentissage plus efficace que le conflit cognitif. 
Car au lieu de confronter les représentations initiales des élèves sur un sujet donné aux connaissances scientifiquement validées, il est préférable de placer l'apprenant face à des situations-problèmes, dans la position d'émettre des réponses incertaines (Turmel et al., 2011). En effet, le doute induirait chez l'apprenant une conscience de l'apprentissage qu'il est en train de réaliser, autrement dit, la métacognition. L'exercice du doute, à travers l'exercice philosophique collaboratif, s'inscrit manifestement dans ces mêmes préconisations même si les études expérimentales ne se sont pas encore penchées sur ces contextes de classe.

Le travail présent ne s'inscrit pas dans la lignée de ces études qui tentent d'expliquer le phénomène de doute par des processus cognitifs. Notre approche, et à notre connaissance il n'y a pas des travaux qui s'intéressent expressément de cette manière au doute, est de décrire des conduites langagières en mettant au centre de nos analyses les interactions entre l'individu et l'environnement et entre l'individu et ses pairs.

\subsection{Analyse à l'aide d'outils philosophiques et linguistiques}

L'analyse de l'expression du doute a nécessité au préalable la transcription des paroles de l'animateur et de l'élève, puis un travail d'annotation (réalisé avec un logiciel de traitement multimodal, ELAN. https://tla.mpi.nl/tools/tla-tools/elan/) et d'analyse à l'aide de critères que nous avons pu définir à partir des références théoriques qui ont été développées dans le paragraphe précédent. Nous avons tenté de combiner des outils d'analyse philosophique avec des outils d'analyse linguistique.

Portant intérêt aux rapports entre la pensée et le langage, nous avons procédé à la mise en place de différents tamis qui permettraient de filtrer les expressions du doute. Le modèle élaboré à partir de ces éléments invite l'analyste à regarder progressivement : des actes de pensée appelés aussi « habiletés de pensée » (Lipman, 2006), qui composent l'acte de philosopher dans lequel sont engagés les participants, et au nombre desquels figurent les habiletés à raisonner, à rechercher, à conceptualiser, à organiser l'information, à communiquer, à s'exprimer, à interpréter (Sasseville \& Gagnon, 2007 : 13) ; la prise de position ou le PDV exprimé par un participant, qui, selon la définition proposée par Rabatel (1998) n'est autre que l'expression linguistique d'une perception subjective, en cela qu'elle rend compte d'une opinion particulière, qui manifeste à la fois la saisie des objets du monde et la façon dont le sujet les saisit; les actes de langage produits lors de ces mouvements philosophiques, le doute se situant quelque part au croisement des directifs, exclamatifs et assertifs ; les marqueurs syntaxiques qui accompagnent dans le discours la réalisation de ces actes (marqueurs lexicaux : verbes, adverbes, semi-auxiliaires-modaux ; morphologiques : modes verbaux etc.) qui nous amènent à examiner la manière dont les participants expriment leur idées. Et pour la dimension interactionnelle, nous nous sommes intéressée principalement aux rôles joués par les participants dans les actes argumentatifs (qui sont principalement, la verbalisation d'une problématique ainsi que la verbalisation d'un argument ou d'un contre-argument).

Précisons que pour cette analyse, curieux avant tout de découvrir comment les élèves s'emparent du doute lorsqu'ils participent à la recherche philosophique sous-tendue par la discussion, nous avons choisi de nous concentrer sur leurs conduites langagières.

Le tableau ci-après reprend et développe les éléments d'analyse qui viennent d'être présentés.

Tableau 1 : Eléments pour un modèle d'analyse du doute

$$
\text { ACTE DE PHILOSOPHER Conceptualiser - Problématiser - Argumenter ; }
$$

(Tozzi, 2007)

$\begin{array}{ll}\text { HABILETE DE PENSEE } & \text { Douter / Hésiter / Reconnaître l'ignorance / S'autocorriger (D1) } \\ \text { (Sasseville \& Gagnon, 2007) } & \text { Questionner / Problématiser / Chercher /Complexifier / (D2) } \\ & \text { Nuancer / Contredire / Contextualiser / Contre-exemple (D3) } \\ & \text { Autres : Exemple / Raison / Critère / Evaluer une raison / Formuler une opinion / } \\ & \text { Distinguer / Clarifier / Comparer / Définir / Reformuler / Formuler une hypothèse }\end{array}$




\begin{tabular}{ll}
\hline $\begin{array}{l}\text { POINT DE VUE } \\
\text { et posture énonciative } \\
\text { (Vion, 2012, Rabatel, 2003) }\end{array}$ & $\begin{array}{l}\text { PDV non explicite / PDV justifié / PDV qui s'oppose / PDV nuancé / PDV repris / } \\
\text { PDV suspendu /PDV co-énoncé etc. ; }\end{array}$ \\
MODALISATION & Eléments lexicaux : \\
& verbes modaux : savoir, penser, douter, ignorer etc.; pouvoir, devoir, vouloir ; \\
& $\begin{array}{l}\text { adverbes modaux épistémiques : certainement, peut-être, probablement, vraiment } \\
\text { etc. ; semi-auxiliaires modaux : pouvoir, devoir... }\end{array}$ \\
& locutions adverbiales : sans doute, bien-sûr etc. ; \\
& proposition en incise : je n'en sais rien, j'en doute \\
& Marques morphologiques : modes verbaux : conditionnel ; \\
& Ratures orales - Hésitations - Silences - Intonation - Mimique ; \\
\hline POLES ARGUMENTATIFS & Proposition - Opposition - Restriction - Tiers (problématisation, « doute ouvert »). \\
\hline (Plantin, 2005) &
\end{tabular}

\subsection{Thématique et spécificités de la discussion}

Avant de procéder à l'analyse conceptuelle et linguistique, il nous semble important de revenir sur la thématique de la discussion ainsi que sur les spécificités discursives et interactionnelles de celle-ci. Dans une approche empirique et interactionniste, la prise en compte des spécificités du dispositif peut permettre par exemple d'évaluer la réalisation ou non des conditions préalables d'une coopération et d'une confrontation entre des PDV.

Il s'agit d'une part d'une discussion très réglée dans laquelle les participants ont des rôles précis : président, réformulateur, synthétiseurs, observateur, discutant. Voici comme introduit l'animateur l'activité et s'assure que chacun comprend son rôle : "avant que nous commencions donc peut-être cette discussion/c'est intéressant qu'on voit un petit peu donc la situation disons dans laquelle nous sommes car euh: ici tout le monde ne fait pas exactement la même chose et n'a pas euh exactement disons la même la même fonction alors euh Mathéo: euh est donc président alors tu peux nous dire euh Mathéo quelle est ta: qu'est-ce que tu fais toi quelle est ton métier finalement ». La discussion est organisée selon un système de tours de parole par attribution externe (c'est le rôle du président). Il y presque pas de silences ou de chevauchements de parole.

D'autre part, l'espace où le locuteur développe son intervention, la scène, se forme davantage par une distribution de tours à des locuteurs particuliers - floor-sharing (Coates, 1994) que par le partage de prises de positions (collaborative floor). Cela s'explique d'une part par la fonction de gestion de la parole associée à un rôle mais aussi par la longueur et le caractère très élaboré des prises de paroles avec la présence, durant la discussion enregistrée, de plusieurs épisodes de mini-entretiens entre l'animateur et un élève.

Les métadonnées nous fournissent des informations également sur la dynamique de la discussion : malgré un aspect fortement argumentatif caractérisant les interventions (les élèves justifient beaucoup leur PDV, apportent des exemples etc.), presque aucun échange n'expose une confrontation directe entre un Proposant et un Opposant (Plantin, 2005) mais il est le plus souvent médié par l'animateur. En effet c'est l'animateur qui met en scène les remises en questions essentiellement par de nombreux épisodes de reformulation, comme dans l'extrait suivant : "c'est-à-dire est-ce que quelqu'un soutiendrait une thèse contraire à donc à celle de Lou heu donc heu reformulée par heu par Thomas à savoir que quand c'est la nature y a pas d'injustice puisque c'est naturel finalement? »" 
Quant à la thématique de la discussion, il faut distinguer deux parties qui la composent et qui sont marquées clairement dans le déroulement discursif. La première partie a comme point de départ la question "pourquoi on dit c'est pas juste? ", alors que la seconde propose un exercice de réflexion à partir d'une situation concrète de partage de gâteau : "quelle est la manière la plus JUSTE de couper un gâteau ? ». Dans la première, les élèves sont incités à argumenter, à chercher des exemples et des raisons, alors que dans la deuxième ils sont invités à faire des propositions pour partager le gâteau et à expliquer leur choix. Dans notre étude, on pourrait s'attendre à trouver plus d'occurrences du doute liées à l'argumentation dans la première partie, que dans la seconde, et des occurrences du doute liées à la problématisation plus nombreuses dans la seconde partie que dans la première. C'est une hypothèse qui nous allons confronter aux données du corpus.

\section{Résultats}

\subsection{Caractéristiques générales de la discussion}

Le tableau ci-après présente quelques caractéristiques générales de la discussion. Nous avons utilisé l'abréviation TP pour « tour de parole »:

Tableau 2 : Caractéristiques générales de la discussion

\begin{tabular}{lccc}
\hline & Partie 1 & Partie 2 & Partie 1+2 \\
\hline Durée totale discussion (mn) & 34.91 & 49.04 & 83.95 \\
Durée dispositif & 35.65 & 34.25 & 69.90 \\
Durée interventions animateur & 17.48 & 19.33 & 36.81 \\
Durée interventions élèves & 17.21 & 29.63 & 46.84 \\
Nb TP & 178 & 244 & 422 \\
Nb TP animateur & 79 & 112 & 191 \\
Nb TP élèves & 99 & 132 & 231 \\
\hline
\end{tabular}

Précisons que dans la durée totale de la discussion ont été comptabilisées : les considérations générales d'introduction de la démonstration avec la prise de parole de l'enseignant accompagnant le groupe ; l'introduction par l'animateur du sujet de la discussion ; la phase d'installation des élèves dans leur rôle et l'explicitation de chaque rôle, en début de chaque partie de la discussion (il y a changement de rôles avant le démarrage de la seconde partie) ; l'analyse faite par les élèves de l'ensemble de la discussion; et enfin l'intervention finale de l'enseignant. Si l'on prend en compte uniquement la durée du dispositif proprement dit, nous observons que les deux parties ont une durée presque égale, ce qui dénote un partage équitable du temps alloué aux deux questions traitées. Cette observation est importante pour les comparaisons futures entre les variables (tour de parole, habiletés de pensée, acte de douter etc.), selon le type d'exercice effectué.

Le graphique ci-après nous montre comment se distribuent les TP selon les locuteurs. Sur l'ensemble de la discussion, presque la moitié du nombre de TP sont attribués à l'animateur (45\%), l'autre moitié se distribue à tous les élèves mais pas avec la même proportion : de $1 \%$ pour les moins bavardes à $9 \%$ pour ceux qui sont intervenus le plus souvent (graphique 1). Selon que l'on est dans l'une ou l'autre des parties de la discussion, la distribution des TP reste assez similaire avec un nombre important d'interventions de la part de l'animateur. A noter que dans chaque partie, l'élève qui est le plus intervenu est celui qui tient le rôle de président, chargé de distribuer la parole à ses pairs. La visualisation nous permet de voir que la gestion de la discussion passe essentiellement par l'animateur qui reformule et met régulièrement en lien les interventions des élèves.

Graphique 1 : L'ensemble de TP de la discussion, selon les locuteurs 


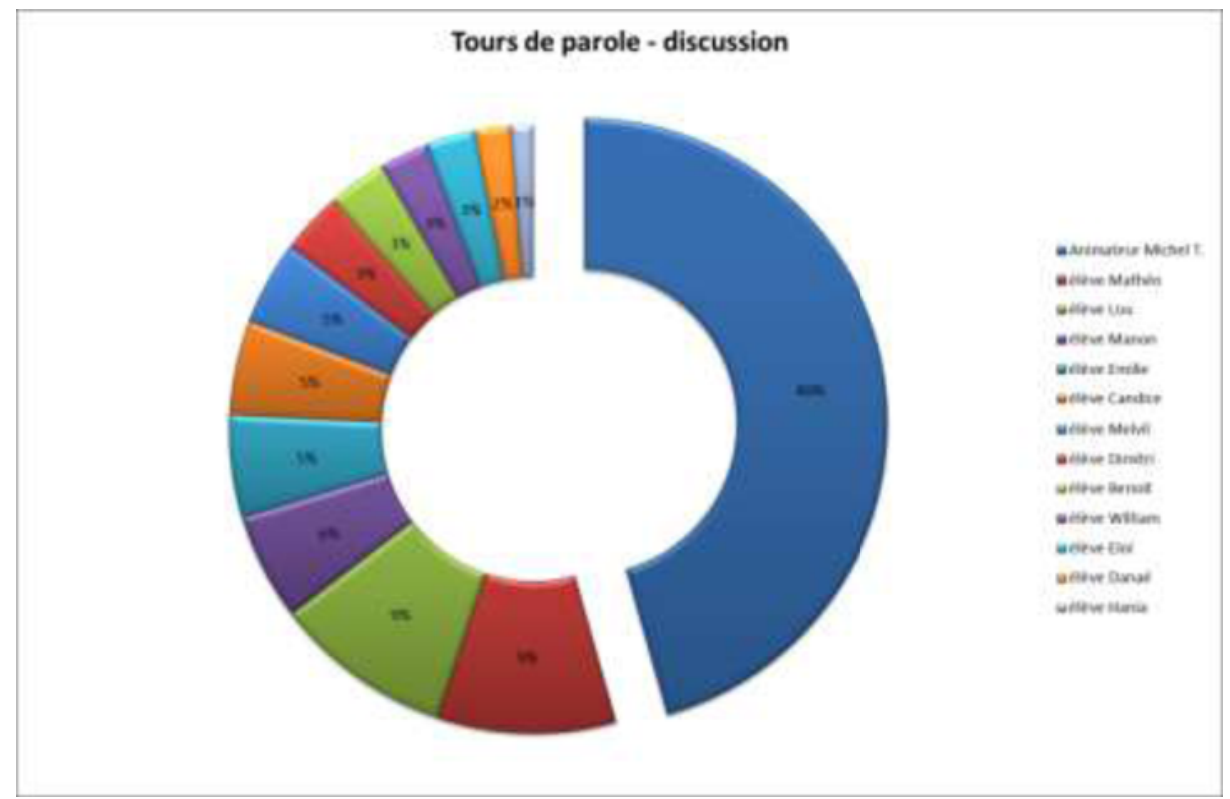

\subsection{Mesures spécifiques}

Pour mieux se représenter les événements (discursifs, cognitifs, dialogiques et langagiers) de la discussion en lien avec l'activité de douter, nous disposons des mesures suivantes: le nombre total de TP relevés lors de la discussion, concernant les élèves ; le nombre total de point de vue (PDV) exprimés par les élèves ; le nombre des TP qui contiennent un ou des PDV; la distribution de ces TP avec PDV dans l'ensemble des TP des élèves ; le nombre total d'habiletés de pensée (HP) mobilisées par les élèves dans la discussion ; la distribution des HP dans l'ensemble des TP qui contiennent un ou des PDV; le nombre d'actes de douter (AD) et selon le type de doute (D1, D2 et D3), dans l'ensemble des HP mobilisées ; la distribution des actes liés au doute dans l'ensemble des TP avec PDV; le nombre d'éléments de modalisation (M) et leur ratio par rapport au nombre de TP avec PDV; enfin le nombre de rôles argumentatifs (RA) exercés par les élèves dans l'interaction.

\subsubsection{Habiletés de pensée et actes associés au doute}

Les mesures ci-dessous (Tableau 3) montrent qu'1 tour de parole d'élève sur 4 exprime un PDV. Tous les TP ne contiennent pas des PDV, c'est le cas des réponses positives («oui ») ou négatives («non »); des confirmations («c'est ça»); des actes de langage simples («je ne sais pas »); lorsque l'élève se voit attribué le rôle de distribuer la parole et nomme le prochain locuteur («je vais donner la parole à... »); des interpellations faites par ceux-ci («est-ce que tu as quelque chose à dire ?») ; les TP qui se réduisent à des hésitations ("parce que enfin», «heu») etc.. Observons également qu'il existe des TP qui contiennent plus d'un PDV et c'est, en effet, le cas de deux interventions que nous allons présenter dans les illustrations.

Tableau 3 : Présence de PDV dans la discussion

\begin{tabular}{lcccc}
\hline & $\begin{array}{c}\text { Nb TP } \\
\text { élèves }\end{array}$ & Nb & Nb & Ratio \\
& TP avec PDV & TP avec PDV/TP \\
\hline $\begin{array}{l}\text { Discussion } \\
\text { partie 1+2) }\end{array}$ & $\mathbf{2 3 1}$ & 63 & 61 & $\mathbf{0 . 2 6}$ \\
\hline
\end{tabular}


Comme certains TP ne contiennent aucune habileté de pensée, nous avons choisi de rapporter le nombre d'habiletés de pensée aux nombre d'interventions qui expriment un PDV. Comme présenté dans le Tableau 4, un élève mobilise en moyenne 3 habiletés de pensée dans une intervention où il exprime un PDV. Parmi de nombreuses autres habiletés on retrouve le doute et les actes associés au doute que nous regroupons ainsi : hésiter - douter -reconnaître l'ignorance - s'autocorriger (D1) ; nuancer-contredire - contre-exemple - contextualiser (D2) ; questionner - problématiser - chercher - complexifier (D3). Nous observons également qu'environ 1 acte sur 3 est un acte de doute ou associé au doute (sixième colonne). Cela montre un certain degré non négligeable de problématisation et de remise en question présentes dans les prises de position des élèves.

Tableau 4 : Le nombre d'habiletés de pensée dont des actes de doute, dans la discussion

\begin{tabular}{lccccc}
\hline & $\begin{array}{c}\text { Nb TP avec } \\
\text { PDV }\end{array}$ & Nb HP & Ratio HP/ & Nb AD & Ratio \\
\hline $\begin{array}{l}\text { Discussion } \\
\text { (partie 1 }+2)\end{array}$ & 61 & 193 & 3.16 & 63 & AD $/ H P$ \\
\hline
\end{tabular}

En détaillant les trois types de doute mobilisés par les élèves, selon l'exercice qu'ils réalisent, nous obtenons les résultats suivants :

Tableau 5 : Les actes de doute, selon le type d'exercice

\begin{tabular}{cccccc}
\hline & Nb HP & Total AD & & Nb AD & \\
\hline & & & D1 & D2 & D3 \\
Partie 1 & $\mathbf{1 0 6}$ & 35 & 7 & 18 & $\mathbf{1 0}$ \\
Partie 2 & $\mathbf{8 7}$ & $\mathbf{2 8}$ & $\mathbf{1 4}$ & $\mathbf{9}$ & $\mathbf{5}$ \\
Partie 1+2 & $\mathbf{1 9 3}$ & $\mathbf{6 3}$ & $\mathbf{2 1}$ & $\mathbf{2 7}$ & $\mathbf{1 5}$ \\
\hline
\end{tabular}

Nous observons que dans la première partie de la discussion les actes d'opposition ou de restriction (D2) et ceux exprimant un doute ouvert (D3) sont plus nombreux dans la première partie que dans la seconde (le double); alors qu'inversement, le sens premier du doute (D1) est davantage présent dans la seconde partie (deux fois plus que dans la première) devançant les deux autres types de doute. Nous avions fait plus tôt l'hypothèse qu'il y aurait plus d'activité argumentative et par conséquent plus de D2 dans la première partie de la discussion par rapport à la seconde. En effet, si l'on considère D3 comme faisant partie de l'activité argumentative, notre hypothèse se confirme par ces résultats. La seconde partie, en revanche, ne se caractériserait pas par plus de doute ouvert (D3), comme nous l'avons supposé, mais par une présence importante d'hésitation, d'absence de certitude et d'auto-remise en question. Cela peut nous rendre attentif à une distinction à opérer entre une auto-interrogation ou questionnement de sens (l'acception de la problématisation, selon M. Tozzi), qui serait spécifique à une situation d'énonciation, et une problématisation en contexte argumentatif, lié davantage à la co-énonciation.

\subsubsection{Modalisation}

Nous avons observé que pour formuler une opinion et entrer dans une activité de justification, les participants s'appuient sur la modalisation. En effet, ils expriment leur attitude par rapport à ce qu'ils énoncent :: «bah:: je pense que y a un p(e)tit peu de l'injustice » (TP 157); « donc *chpense que:: bah même si quelqu'un travaille mal // heu bah i(l) devrait avoir autant de nourriture que tout le monde » (TP 318) ; mais aussi leur attitude par rapport à leur propre énonciation : «i(l) y a un exemple mais je l'ai pas j'l'ai pas passé quoi c'est pas vrai » (TP95); « on le pense vraiment //(en)fin on le pense peut-être mais pas beaucoup beaucoup » (TP 68). Les deux types de modalisation peuvent coexister dans la même intervention: «... c'est vrai que les personnes // si elles l'auraient su elles l'auraient (x) donc y aurait peut-être personne de mort mais heu $\mathrm{j}(\mathrm{e})$ trouve que la nature c'est en fait eh ben c'est comme l'homme sauf que heu c'est pas la même chose » (TP 169). 
Les mesures effectuées nous montrent (cf. Tableau 6) que les élèves utilisent en moyenne 3 modalisateurs dans une intervention qui contient un PDV. Selon les parties, on serait à 2 modalisateurs par intervention dans la première partie et à 4 modalisateurs dans la seconde. Les interventions des élèves sont généralement longues, ce qui laisserait suffisamment d'espace à l'expression de la subjectivité (modalisation), mais peut-être au détriment d'une dynamique interactionnelle.

Tableau 6 : La modalisation, selon le type d'exercice

\begin{tabular}{lccc}
\hline Discussion & TP avec PDV & Nb. M & $\begin{array}{c}\text { Ratio } M \\
\text { /TP avec PDV }\end{array}$ \\
\hline Partie 1 & $\mathbf{3 7}$ & $\mathbf{7 9}$ & $\mathbf{2 , 1 3}$ \\
Partie 2 & $\mathbf{2 4}$ & $\mathbf{9 5}$ & $\mathbf{3 , 9 5}$ \\
Partie 1+2 & $\mathbf{6 1}$ & $\mathbf{1 7 4}$ & $\mathbf{2 . 8 5}$ \\
\hline
\end{tabular}

Parmi les formes les plus fréquentes de modalisation on compte les verbes d'opinion («je crois », «je trouve », «je pense ») dont des verbes qui expriment clairement le doute ou l'absence de certitude («je sais pas trop », "je crois», "je trouve que», "il me semble que »). Il y a ensuite des adverbes modalisateurs («normalement», «sûrement», «justement», «vraiment») avec des formes qui expriment clairement le doute («peut-être », «un peu», «pas forcément»). Les élèves utilisent également les semi-auxiliaires modaux («on peut avoir», "ça peut être», "on peut pas faire autrement», "on peut pas le savoir ») ainsi que le mode conditionnel pour envisager les choses sous l'angle de la possibilité plutôt que sous l'angle de la certitude.

\subsubsection{Rôles actantiels argumentatifs}

Nous avons observé la distribution des rôles actantiels (Proposant $-\mathrm{P}$; Opposant $-\mathrm{O}$; Tiers $-\mathrm{T}$ ) dans la discussion selon le type de rôle et selon l'exercice réalisé. Les rôles argumentatifs s'exprimant à l'aide des actes de pensée, nous avons regardé leur présence par rapport à l'ensemble d'habiletés (exprimer une opinion, donner une raison, un exemple ou un contre-exemple, problématiser etc.)

Tableau 7 : Distribution des rôles argumentatifs, selon le type d'exercice

\begin{tabular}{|c|c|c|c|c|c|c|}
\hline \multirow{2}{*}{ Discussion } & \multirow[t]{2}{*}{$\mathrm{Nb} \mathrm{HP}$} & \multicolumn{3}{|c|}{$N b R A$} & \multirow[t]{2}{*}{ Total RA } & \multirow{2}{*}{$\begin{array}{c}\text { Ratio } \\
\text { RA/HP }\end{array}$} \\
\hline & & $P$ & $O$ & $T$ & & \\
\hline Partie 1 & 106 & 24 & 14 & 7 & 45 & 0,42 \\
\hline Partie 2 & 87 & 14 & 15 & $\mathbf{0}$ & 29 & $\mathbf{0 , 3 3}$ \\
\hline Partie $1+2$ & 193 & 38 & 29 & 7 & 74 & $\mathbf{0 , 3 8}$ \\
\hline
\end{tabular}

Dans la première partie de la discussion, l'ensemble de rôles argumentatifs sont plus nombreux que dans la deuxième (45 contre 29). On voit qu'environ 1 acte sur 2,5 participe à l'expression d'un rôle actantiel dans la première partie où les postures de Proposant sont les plus nombreuses. A remarquer que tous les rôles qui peuvent être attribués au Tiers se trouvent dans la première partie, ce qui nous laisserait supposer que le doute ouvert ou la problématisation sont lié directement à l'activité argumentative (spécifique de la première partie).

En résumé, il y aurait dans la première partie, où les élèves sont invités à justifier, plus d'expressions du doute lié à l'activité argumentative (problématisation et remise en question des PDV) et donc à la coénonciation. En revanche, la seconde partie, dans laquelle les élèves font des propositions pour le partage, se caractérise par la présence de l'incertitude exprimée au niveau de l'énonciation, et également par une présence plus importante de modalisation. 


\title{
6 Illustrations
}

\subsection{Evolution des PDV}

A titre d'illustration, nous présentons d'abord les deux interventions contenant deux PDV. Lou recherche des raisons à la question posée par l'animateur : «pourquoi on dit c'est pas juste ?» :

\begin{abstract}
TP68 : Lou : ben moi je pense que c'est plutôt pour l'injustice (en)fin:: des fois i(1) y a des injustices par exemple heu justement l'exemple de Melvil il était bien (en)fin::: // des fois $i(1) y$ a des frères et des sœurs qui ont des injustices mais des fois c'est toi qui a/ qui a plus de chance et des fois c'est l'autre qui a plus de chance donc des fois on dit c'est pas juste // mais (en)fin on le pense vraiment // (en)fin on le pense peut-être mais pas beaucoup beaucoup
\end{abstract}

L'élève propose d'abord un PDV qui s'appuie sur la raison de l'existence, dans certains cas (« des fois ») de l'injustice, pour qu'à la fin de sa prise de parole elle accentue à la fois la certitude avec laquelle on pense cette injustice ( «vraiment ») et le fait que celle-ci ne résiste pas à l'examen de la réflexion («on le pense peut-être mais pas beaucoup beaucoup »). Elle propose donc un deuxième PDV qui problématise le concept d'injustice. On observe d'ailleurs comment la modalisation participe à ce processus de réflexion, car le "je pense » utilisé initialement pour exprimer une opinion devient plus loin "on le pense » pour exprimer un énoncé à portée générale à partir d'un cas particulier, celui des frères et des sœurs qui subissent chacun leur tour l'injustice. Mais le « on » introduit ici un énoncé qui est plutôt pris en charge par la locutrice, s'agissant d'un «je » ou d'un «nous » engagés. L'adverbe de modalisation, «vraiment», appuie l'idée de la présence du locuteur qui, en réalité, ne fait autre chose que mettre en question son PDV initial. En employant l'adverbe modalisateur du doute, "peut-être », Lou exprime cette opposition par l'utilisation du connecteur restrictif « mais », et à nouveau une modalité, cette fois à l'aide d'un quantificateur restrictif, « pas beaucoup beaucoup », dont la répétition augmente l'effet visé.

Le deuxième exemple se prête à une analyse similaire. Voici l'intervention de Candice :

TP 85 : Candice : moi je pense qu'(il) y a plusieurs (en)fin heu // plusieurs sens de c'est pas juste parce que y a / heu (il) y a // c'est pas juste qui peut créer un conflit par exemple heu qui est vraiment dans l'inégalité heu // par exemple heu : // je sais pas trop \{rires\} // (il) y a aussi heu le c'est pas juste bah un peu heu // capricieux on va dire // (en)fin qu'on pense pas trop // qui est plutôt heu heu c'est pas juste heu mais en fait c'est juste (en )fin heu voilà et ben après heu ça dépend des situations mais :

Dans cette intervention, l'élève mobilise de nombreuses habiletés de pensée : la formulation d'une opinion («je pense ») par une nuance apportée à la notion d'injuste. Elle distingue entre un effet d'injustice et un caprice. La recherche d'un exemple, même si elle ne le trouve pas sur le moment, ce qui lui vaut une reconnaissance de son ignorance, est suivie d'une définition du mot «capricieux » (" on va dire // (en)fin qu'on pense pas trop »). Le PDV nuancé initial se transforme en une vraie problématique qui l'amène à une expression paradoxale («c'est pas juste mais en fait c'est juste ») qui, pour être comprise, demande d'être contextualisée ( «ça dépend des situations »). Les deux illustrations analysées combinent une restriction et une problématisation, le rôle de Tiers se préfigurant à chaque fois dans la logique argumentative des échanges.

\subsection{Rôles argumentatifs et expressions du doute}

Dans les illustrations ci-après, nous analysons deux interventions de la seconde partie de la discussion, pour voir comment se croisent les rôles argumentatifs et l'expression du doute. Au problème du partage du gâteau, un élève propose une solution inédite et surprenante : partager le gâteau en parts égales et, s'il y a quelque chose qui est particulièrement bon sur le gâteau (caramel, chocolat ou chantilly), on le donne au chien pour éviter toute injustice. Emilie, qui n'est pas d'accord avec cette solution, se positionne ainsi :

TP 241 : Emilie : bah moi * chpense pas que c'est une très bonne idée heu de

TP 242 : Animateur : donc tu es pas d'accord ? 


\begin{abstract}
TP 243 : Emilie : oui voilà // parce que (en)fin *chpense que bah:: la part où y a plein de caramel de/ (en)fin * chpense qu'on devrait couper les part égales normalement // et que bah tant pis pour le (en)fin * chais pas on donne les parts heu normalement aux personnes et:: et voilà dans l'ordre où elles sont tout ça et on va pas (en)fin on va pas marcher heu:: à l'autre bout de la table pour donner la meilleure part à quelqu'un on la donne à la personne qui est en face de nous et voilà // donc si
\end{abstract}

Emilie évalue la proposition faite auparavant et doute de sa pertinence («*chpense pas que c'est une très bonne idée »). La reformulation de l'animateur ("donc tu n'es pas d'accord ») lui permet de confirmer son opposition («oui voilà»). Elle s'affirme donc comme Opposant et tout de suite comme Proposant en apportant sa réponse : "*chpense qu'on devrait couper les part égales normalement // et que bah tant pis pour le » - on comprend qu'il s'agit de la garniture, point sur lequel elle reviendra dans le tour suivant. Emilie poursuit en proposant un critère pour partager : comme ça vient, " dans l'ordre ». Elle évalue la position contraire de la sienne (privilégier certains en leur donnant les meilleures parts): «on va pas (en)fin on va pas marcher heu:: à l'autre bout de la table pour donner la meilleure part à quelqu'un ». On observe qu'elle utilise le conditionnel (« on devrait couper ») ou des expressions qui exprime l'absence de certitude ( $*$ *chais pas on donne les parts heu »). L'animateur lui demande de justifier pourquoi il lui semble juste de partager en parts égales, occasion pour Emilie de revenir sur le problème qu'elle essaie de soulever, sans qu'elle soit véritablement comprise :

TP 247 : Emilie : bah:: parce que:: (en)fin // le chocolat et tout ça bah on peut pas en mettre plus d'un côté ou pas (en)fin on essaie d'en mettre un peu // partout et bah après * chpense que c'est bien coupé normalement donc même si y a un endroit où y en a un peu plus c'est pas grave vu que à la base c'est un gâteau et c'est pas obligé (en)fin y a des gâteaux où y a rien $\mathrm{d}(\mathrm{e})$ ssus $\mathrm{i}(\mathrm{ls})$ sont natures donc ça sert à rien de vouloir une part où $\mathrm{y}$ a plein $\mathrm{d}(\mathrm{e})$ choses

L'élève renforce graduellement son opposition en évaluant la raison qu'elle donne et en faisant remarquer que la garniture n'est pas importante ; enfin elle finit par apporter la raison déterminante selon laquelle un gâteau c'est un gâteau et c'est ça qui est essentiel, le reste n'est qu'accessoire («ça sert à rien de vouloir une part où y a plein $\mathrm{d}(\mathrm{e})$ choses $»)$. Dans sa posture d'Opposant, Emilie remet en question la solution proposée par Melvil mais non pas pour le convaincre qu'il a tort. Emilie ne prend pas véritablement le rôle d'un Tiers, car elle prend position et assume son PDV. D'ailleurs elle utilise de nombreux modalisateurs, que nous avons soulignés dans les citations. L'élève soulève une problématique et s'engage dans une démarche qui questionne mais qui n'est pas reconnu comme tel dans la logique des échanges.

Cette illustration nous montre le passage presque imminent d'un doute vécu sous l'angle de l'incertitude énonciative à un doute engagé dans la situation argumentative conduisant à la problématisation.

\title{
7 Conclusion
}

Il nous semble indispensable d'analyser d'autres extraits pour mieux observer comment le doute peut être articulé avec les actes philosophiques d'argumentation et de problématisation.

Arrivée au terme de cette analyse, nous proposons une esquisse de modèle d'observation du doute dans un contexte interactionnel (Tableau 8). Nous remarquons qu'il y a deux acceptions principale du doute : questionner - correspondant aux cases 1 et 6 - et remettre en question (contredire, s'opposer, formuler une restriction) - les cases 2, 4, 5. La case 3 correspond quant à elle à une posture sceptique, difficilement défendable. Le doute individuel (1) se définit principalement dans un contexte d'énonciation et se manifeste comme un questionnement de sens, un embarras, alors que le doute ouvert ou la problématisation (6) est directement lié à un questionnement en situation co-énonciative et argumentative, qui n'est pas lui-même une argumentation mais l'évaluation des arguments. Ce modèle doit être testé sur d'autres discussions philosophiques, en vue d'être amélioré et validé. 
Tableau 8 : Modèle d'analyse du doute en contexte dialogique et interactionnel

\begin{tabular}{|c|c|c|c|}
\hline $\begin{array}{c}\text { Contexte dialogique et } \\
\text { interactionnel }\end{array}$ & Proposant & Opposant & Tiers \\
\hline $\begin{array}{c}\text { Attitude du locuteur en } \\
\text { énonciation }\end{array}$ & $\begin{array}{c}\text { DOUTE } \\
\text { (1) } \\
\text { Hésitation /Absence de } \\
\text { certitude/ Possibilité }\end{array}$ & $\begin{array}{l}\text { AUTOCRITIQUE } \\
\text { (2) } \\
\text { Mise en question de sa } \\
\text { propre proposition }\end{array}$ & $\begin{array}{c}\text { SUSPENSION DU } \\
\text { JUGEMENT } \\
(3) \\
\text { Suspension assentiment à } \\
\text { une proposition }\end{array}$ \\
\hline $\begin{array}{l}\text { Attitude du locuteur } \\
\text { enco-énonciation }\end{array}$ & $\begin{array}{c}\text { RESTRICTION } \\
(4) \\
\text { Mise en question d'une } \\
\text { autre proposition } \\
\text { (Proposant } \rightarrow \text { Opposant) }\end{array}$ & $\begin{array}{c}\text { RESTRICTION } \\
(5) \\
\text { Mise en question d'une } \\
\text { autre proposition }\end{array}$ & $\begin{array}{c}\text { PROBLEMATISATION } \\
\text { (6) } \\
\text { Evaluation des arguments } \\
\text { Doute ouvert }\end{array}$ \\
\hline
\end{tabular}

\section{Références bibliographiques}

Coates, J. (1994) : No gap, lots of overlap : Turn-taking patterns in the talk of women friends . In D. Graddol,

J. Maybin et B. Stieter (éds). Researching Language and Literacy in Social Context, 177-192. Clevedon, Open University.

Crahay, M. \& Fagnant, A. (2007). A propos de l'épistémologie personnelle : un état des recherches anglo-saxonnes (note de synthèse). Revue française de pédagogie, $n^{\circ} 161 /$ oct.-déc., 79-117.

De Nuchèze, V. \& Colletta, J.M. (eds.) (2002). Guide terminologique pour l'analyse des discours. Bern : Peter Lang.

Dewey, J. (1910, 1997). How We Think. New York: Dover Publications, INC, Mineola.

Dubois, J. et al., (1973). Dictionnaire de Linguistique. Modalisation. Paris : Larousse.

Ducrot, (1984). Le dire et le dit. Paris : Editions de Minuit.

Houdé, O. (2014). Le raisonnement. Que sais-je ? Paris : PUF.

Lipman, M. (2003). Thinking in Education. 2nd Edition. Cambridge.

Lipman, M. (2006). A l'Ecole de la pensée (tr. N. Decostre). Bruxelles : Editions De Boeck Université.

Mondada, L. (1999). L'organisation séquentielle des ressources linguistiques dans l'élaboration collective des descriptions. Langage et Société, n 89, septembre 1999, 9-36.

Peirce, C. S. (1877). The Fixation of Belief in Popular Science Monthly 12 (November 1877). Repéré le 10 novembre 2015 à http://www.peirce.org/writings/p107.html

Peirce, C. S. (1958). Collected Papers. Vols. 1-6. Cambridge (Mass.): Harvard University Press.

Plantin, C. (2005). L'argumentation: histoire, théories et perspectives. Que Sais-Je ? Paris : PUF.

Plantin, C. (2015). Dictionnaire de l'argumentation. Lyon : ENS Éditions.

Potvin, P., Masson, S. et Riopel, R. (2009, 12 mai). Étude neuroscientifique du rôle du doute dans les apprentissages scientifiques qui nécessitent des changements conceptuels. Communication présentée dans le cadre du $77 \mathrm{e}$ Congrès de l'ACFAS, Université d'Ottawa, Ottawa.

Rabatel, A. (2003). Le point de vue, entre langue et discours, description et interprétation : état de l'art et perspectives. Cahiers de praxématique, Publications de l'Université Paul Valéry, 7-23.

Rabatel, A. (1998). La Construction textuelle du point de vue. Lausanne-Paris : Delachaux \& Niestlé.

Sasseville, M \& Gagnon, M, (2007). Penser ensemble à l'école. Québec : Les Presses de l'Université de Laval (coll. « Dialoguer »).

Tiercelin, C. (2005). Le doute en question. Paris : Editions de l'Eclat.

Turmel, É. (2012). L'incertitude comme nouvelle piste pour favoriser le changement conceptuel lors de l'apprentissage de notions scientifiques. In Spectre, 42(1), 22-25. 
Tozzi, T. Quelles compétences développent les discussions à visée philosophique (dvp) chez les élèves? Diotime, $\mathrm{n}^{\circ} 33$ (avril 2007). Repéré le 12 novembre 2015 à http://www.educrevues.fr/Diotime/affichagedocument.aspx?iddoc $=32829 \&$ pos $=281$

Tozzi, M. (2015). La coopération dans la Discussion à Visée Démocratique et Philosophique (DVDP). Diotime, $\mathrm{n}^{\circ} 64$ (avril 2015). Repéré le 19 novembre 2015 à http://www.educrevues.fr/DIOTIME/AffichageDocument.aspx?iddoc=103754\&pos=4\#N1

Vion, R. (2012). La modalisation: un mode paradoxal de prise en charge. Filol. linguíst. port., n 14(2), $203-224$.

Turmel, E., Potvin, P., Masson, S. \& Riopel, M. (2011, mai). Étude neurodidactique du rôle du doute dans les apprentissages scientifiques qui nécessitent des changements conceptuels. Communication présentée au Colloque de neuroéducations (ACFAS), Sherbrooke, Québec. Repéré le 7 décembre 2015 à https://www.youtube.com/watch?v=2i6ceGRM3eU

\footnotetext{
${ }^{\text {i } B e ́ n e ́ f i c i a i r e ~ d ' u n e ~ A l l o c a t i o n ~ d e ~ r e c h e r c h e ~ d e ~ l a ~ R e ́ g i o n ~ R h o ̂ n e-A l p e s ~}$
} 